\title{
Cost-Effectiveness Analysis from a Randomized Controlled Trial of Tailored Exercise Prescription for Women with Breast Cancer with 8-Year Follow-Up
}

\author{
Louisa G. Gordon $1,2,3, * \mathbb{B}$, Elizabeth G. Eakin ${ }^{3}$, Rosalind R. Spence ${ }^{4}$, Christopher Pyke ${ }^{5}$, \\ John Bashford ${ }^{6}$, Christobel Saunders ${ }^{7}$ and Sandra C. Hayes ${ }^{4}$ \\ 1 Population Health Department, QIMR Berghofer Medical Research Institute, Locked Bag 2000, \\ Royal Brisbane Hospital, Brisbane, Qld 4029, Australia \\ 2 School of Nursing, Queensland University of Technology (QUT), Kelvin Grove, Brisbane, Qld 4059, Australia \\ 3 Faculty of Medicine, School of Public Health, The University of Queensland, Herston, Brisbane, Qld 4006, \\ Australia; e.eakin@uq.edu.au \\ 4 Menzies Health Institute Qld, Griffith University, Nathan, Brisbane, Qld 4111, Australia; \\ r.spence@griffith.edu.au (R.R.S.); sandi.hayes@griffith.edu.au (S.C.H.) \\ 5 Mater Public and Private Hospital, South Brisbane, Qld 4101, Australia; Christopher.Pyke@mater.org.au \\ 6 The Wesley Hospital, Auchenflower, Brisbane, Qld 4066, Australia; j.bashford@mac.com \\ 7 Faculty of Health and Medical Sciences, University of Western Australia, Perth, WA 6009, Australia; \\ christobel.saunders@uwa.edu.au \\ * Correspondence: louisa.gordon@qimrberghofer.edu.au
}

Received: 9 November 2020; Accepted: 17 November 2020; Published: 19 November 2020

\begin{abstract}
Studies show conflicting results on whether exercise interventions to improve outcomes for women with breast cancer are cost-effective. We modelled the long-term cost-effectiveness of the Exercise for Health intervention compared with usual care. A lifetime Markov cohort model for women with early breast cancer was constructed taking a societal perspective. Data were obtained from trial, epidemiological, quality of life, and healthcare cost reports. Outcomes were calculated from 5000 Monte Carlo simulations, and one-way and probabilistic sensitivity analyses. Over the cohort's remaining life, the incremental cost for the exercise versus usual care groups were $\$ 7409$ and quality-adjusted life years (QALYs) gained were 0.35 resulting in an incremental cost per QALY ratio of AU $\$ 21,247$ (95\% Uncertainty Interval (UI): Dominant, AU\$31,398). The likelihood that the exercise intervention was cost-effective at acceptable levels was 93.0\%. The incremental cost per life year gained was AU $\$ 8894$ (95\% UI Dominant, AU $\$ 11,769$ ) with a 99.4\% probability of being cost effective. Findings were most sensitive to the probability of recurrence in the exercise and usual care groups, followed by the costs of out-of-pocket expenses and the model starting age. This exercise intervention for women after early-stage breast cancer is cost-effective and would be a sound investment of healthcare resources.
\end{abstract}

Keywords: cost-utility analysis; cost-effectiveness analysis; exercise; breast cancer

\section{Introduction}

Breast cancer is the most common cancer among women worldwide. By 2040, an estimated 3.1 million people will be diagnosed with breast cancer, an increase of $47 \%$ from 2018 [1]. The annual number of deaths worldwide are expected to rise to 991,904 by 2040 [1]. Although 5-year survival rates for localized breast cancer are high at $95 \%$ [2], incidence is also high (one in seven women will develop breast cancer [3]) with key lifestyle risk factors remaining widespread and growing. Risk factors for 
postmenopausal breast cancer include obesity, having fewer children, low levels of physical activity, reduced levels of breastfeeding, and having children later in life [4].

Survivors of breast cancer and its treatment can experience reduced quality of life that lingers well after diagnosis and treatment. Long-term effects can include fatigue, pain, cognitive limitations, menopausal symptoms, depression, fear of recurrence, sleeping problems, and decreased physical fitness [5]. Increased fatigue and decreased physical fitness can lead to longer absences from work and delayed return to everyday activities [6]. Hence, the need to improve the quality of life of breast cancer survivors is especially important as their numbers increase.

Physical activity is advocated by cancer agencies and public health organizations to reduce the impacts of symptoms and side-effects from cancer and its treatment [7-11]. Evidence supports the beneficial effects of physical activity during breast cancer treatment and beyond, particularly when the exercise dose exceeds 150 min of moderate intensity, mixed mode exercise per week [10]. Moreover, by alleviating side-effects and improving recovery, systematic reviews consistently show that physical activity reduces breast cancer mortality and events, and all-cause mortality [12-15]. In 2011, a randomized controlled trial of a tailored exercise intervention, Exercise for Health (EfH), was undertaken in Brisbane, Australia for newly diagnosed women with early-stage breast cancer [16,17]. At 18 months, the intervention group had superior quality of life and fitness and lower fatigue than women in the control group [17]. At 8-years follow-up, significant increases in overall and breast-cancer survival were observed in the intervention group, adjusted for cancer stage [18].

Despite the mounting evidence on the health benefits of exercise interventions for breast cancer, relatively little is known about their economic value to inform decisions about wider uptake into routine care [19]. Six economic evaluations have assessed the costs and effects of physical activity interventions for women with breast cancer [20-25]. While most were based on randomized controlled trials, the majority modelled short-term economic benefits and the findings were contradictory. The purpose of this study is to undertake a cost-effectiveness analysis using long-term 8-year follow-up data and model the expected longer-term consequences of the EfH intervention.

\section{Materials and Methods}

\subsection{Overview}

We undertook a Markov cohort model for the cost-effectiveness analysis and modelled women with early stage breast cancer over their remaining lifetime. The measure of benefit used was quality-adjusted life years (QALYs), a generic metric that combines survival with quality of life, commonly used for economic evaluations. Costs and QALYs were aggregated in yearly cycles and compared across the exercise intervention and usual care strategies. Data inputs were obtained from the EfH-randomized controlled trial, supplemented with epidemiological, quality of life, and healthcare cost studies. We adhered to the Consolidated Health Economic Evaluation Reporting Standards (CHEERS) statement for reporting economic evaluations and good practice guidelines for decision-analytic modelling for healthcare interventions [26,27].

\subsection{Intervention and Comparator}

The exercise intervention was based on the EfH trial conducted between October 2006 and June 2008 in Brisbane, Australia. EfH was a randomized controlled trial evaluating an 8-month exercise program for women after surgery for primary breast cancer. Women were recruited from four hospitals $(n=194)$. On average women were aged 52.4 years (standard deviation 8.5$)$, were overweight (mean body mass index $26.6 \pm 5.2 \mathrm{~kg} / \mathrm{m}^{2}$ ), 92.3\% had Stage I or II disease [28]. Personal and diagnostic characteristics were similar across the groups [28]. Full details are available [28] but briefly, the intervention involved 16 sessions with an exercise physiologist across the 8-month intervention. The aim of the sessions is to support women to be exercising at least four days per week for $45 \mathrm{~min}$, including aerobic and resistance-based exercise, with individual prescriptions tailored to each woman's 
ability. The comparator or "usual care" group received no specific intervention but may have received information related to exercise following breast cancer from health professionals or other resources during the course of their health care [28]. This may have included undertaking exercise on the women's own accord, seeking private professional exercise services or not doing any exercise by preference. All participants gave their informed consent for inclusion before they participated in the study. The study was conducted in accordance with the Declaration of Helsinki, and the EfH trial (ACTRN: 012606000233527) was approved by the Human Research Ethics Committee at the Queensland University of Technology and each of the four participating hospitals.

\subsection{Model Structure}

A Markov cohort model was designed with five mutually exclusive health states (Figure 1) starting in the "early stage I and II breast cancer" health state. Women either remained there or, following disease progression, moved to locoregional or metastatic cancer health states and/or death from breast cancer. At any time, the women could die of other causes. Movement between health states occurred by transition probabilities. Relevant costs and health utilities (similar to quality of life) were assigned to each health state and aggregated over time.

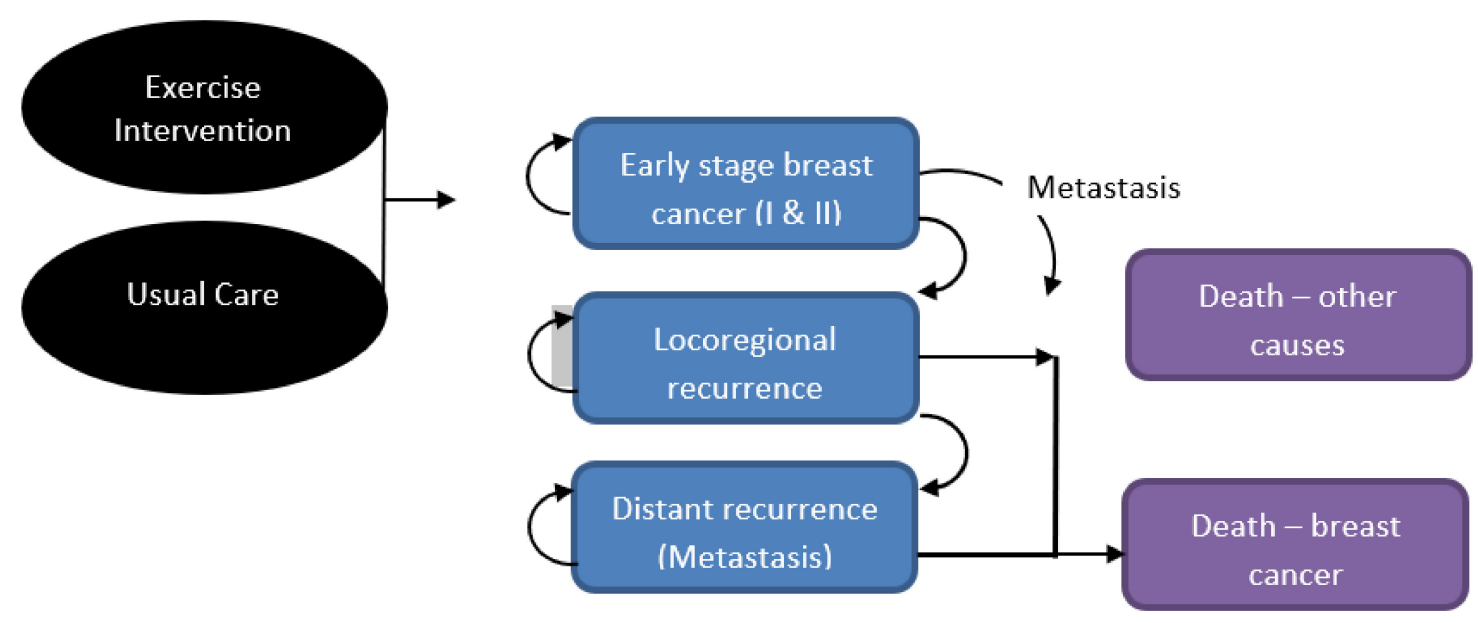

Figure 1. Illustration of Markov model health states.

\subsection{Data Inputs}

Full details of model inputs are summarized in Table 1 and calculations are provided in the Supplementary file. 
Table 1. Model inputs, mean, and sensitivity values and sources.

\begin{tabular}{|c|c|c|c|c|}
\hline Description & Mean & Low & High & Source \\
\hline Age entering the cycle (years) & 52 & 44 & 60 & EfH trial, Hayes 2018 [18] \\
\hline \multicolumn{5}{|l|}{ Annual transition probabilities } \\
\hline Early stage to distant recurrence & 0.018 & 0.016 & 0.019 & Wu 2016 [29] \\
\hline Early stage to local recurrence & 0.007 & 0.006 & 0.007 & As above \\
\hline Local recurrence to distant & 0.0997 & 0.0897 & 0.1097 & Wapnir 2006 [30] \& Anderson 2009 [31] \\
\hline Death from all causes (by age) & Table & \multicolumn{2}{|c|}{ Values differ by age } & Supplementary File, Life tables, female [32] \\
\hline Death from distant recurrence & 0.230 & 0.207 & 0.253 & SEER data [33] \\
\hline Death from local recurrence & 0.069 & 0.006 & 0.007 & Witteveen 2014 [34] \\
\hline \multicolumn{5}{|l|}{ Trial-based probabilities } \\
\hline Non-BrC mortality for exercise intvn & 0.0006 & 0.0000 & 0.0032 & EfH trial, Hayes 2018 [18] \\
\hline Non-BrC mortality for usual care & 0.0047 & 0.0015 & 0.0110 & As above \\
\hline $\mathrm{BrC}$ mortality for exercise intvn & 0.0059 & 0.0028 & 0.0109 & As above \\
\hline BrC mortality for usual care & 0.0096 & 0.0046 & 0.0176 & As above \\
\hline $\mathrm{BrC}$ recurrence in exercise intvn & 0.0072 & 0.0037 & 0.0125 & As above \\
\hline $\mathrm{BrC}$ recurrence in usual care & 0.0076 & 0.0033 & 0.0150 & As above \\
\hline \multicolumn{5}{|l|}{ Costs (AU\$) } \\
\hline Exercise intervention & 1344 & 1209 & 1478 & EfH trial, Gordon 2017 [20] \\
\hline Local recurrence & 8679 & 7811 & 9547 & Verry 2012 [35] \\
\hline Distant recurrence & 27,677 & 24,900 & 30,434 & As above \\
\hline BrC survivors' follow-up care & Table & \multicolumn{2}{|c|}{ Values differ by year post $\mathrm{dx}$} & Supplementary File [36] \\
\hline End-of-life-BrC & 25,475 & 22,928 & 28,023 & Reeve 2017 [37] \\
\hline End of life—other causes & 12,122 & 10,910 & 13,334 & As above \\
\hline Out-of-pocket expenses (annual) & 2538 & 797 & 9079 & Deloitte 2016 [38], first 2 years only \\
\hline \multicolumn{5}{|l|}{ Costs of productivity losses (AU\$) from } \\
\hline Premature death from breast cancer & 149,909 & 134,918 & 164,900 & Carter 2016 [39] \\
\hline Distant recurrence & 34,719 & 31,248 & 38,191 & Deloitte $2016[38,40]$ \\
\hline Local recurrence & 22,785 & 20,506 & 25,063 & As above \\
\hline BrC early stage & 10,850 & 9765 & 11,935 & As above \\
\hline Carers for metastases & 56,419 & 50,777 & 62,061 & As above \\
\hline Carers for locoregional cancer & 29,295 & 26,365 & 32,224 & As above \\
\hline Carers for no recurrence/early stage & 2170 & 1953 & 2387 & As above \\
\hline \multicolumn{5}{|l|}{ Health utilities (quality of life) } \\
\hline Utility for women at baseline & 0.818 & 0.718 & 0.918 & Paracha 2016 [41] \\
\hline Additional utility with exercise program & 0.070 & 0.040 & 0.10 & EfH trial, Gordon 2017 [20], first year only \\
\hline Additional utility with usual care & 0.020 & 0.000 & 0.06 & As above \\
\hline Utility for local recurrence & 0.670 & 0.567 & 0.767 & Paracha 2016 [41] \\
\hline Utility for distant recurrence & 0.640 & 0.540 & 0.74 & “ \\
\hline Utility for terminal BrC & 0.514 & 0.414 & 0.614 & “ \\
\hline
\end{tabular}

$\mathrm{ABS}=$ Australian Bureau of Statistics; $\mathrm{BrC}=$ breast cancer; $\mathrm{dx}=$ diagnosis; EfH = exercise for health; intvn = intervention; SEER = surveillance, epidemiology, and end results. 
Intervention-related variables: Model variables relating to the EfH intervention arm (Table 1 for details) included the additional intervention costs, the probability of all-cause mortality and breast cancer mortality for women in the exercise intervention, recurrence rates, and the health utility gain attributed to the exercise intervention. Parallel variables for the usual care arm were applied. All other variables in the model applied commonly to both comparison arms.

Transition probabilities: The model transition probabilities between the health states and events used data from the EfH trial and published literature of large, long-term, and recent studies (Table 1). Formulas were used to convert rates into one-year probabilities [42]. The age-specific probabilities of death from all causes was derived from the female age-specific mortality rates recorded by the Australian Bureau of Statistics [32]. Locoregional and distant recurrence rates were obtained from a retrospective study conducted by Wu et al. (2016) with a median follow-up of 6.9 years [29]. The probability of dying from breast cancer was calculated using the 10-year survival rate from the study conducted by Witteveen et al. (2015) [34] (women were diagnosed three years earlier than women in the EfH study) and US Surveillance, Epidemiology, and End Results data [33]. The probability of developing metastatic cancer after locoregional disease was a weighted average from node negative and node positive cohorts [30,31].

Health utilities: Utilities are used to calculate QALYs, by applying utility weights (scored from 0 =worst health to $1=$ full health) to the length of life remaining. Health utilities were directly obtained from the EfH trial with improvements reported for both the intervention (0.07) and usual care groups (0.02) over the trial period [20]. These were applied only for the duration of the trial and no assumption of ongoing benefit was made. Utilities for women with breast cancer at pre-diagnosis and at locoregional, metastatic, and terminal disease were extracted from a systematic review of health utilities in breast cancer by Paracha et al. (2016) [41] (Table 1). The pre-diagnosis utility was assigned to the early stage breast cancer health state and the others applied accordingly.

Costs: A societal cost perspective was employed considering healthcare system, patient, and carer economic burdens. Intervention costs are previously detailed in [20] and included professional labour, administration, educational materials, office rental, phone charges for program delivery, equipment, and travel costs (Supplementary File). The costs of breast cancer follow-up care, costs of treatment for recurrence, and productivity losses were included. Follow-up care costs were calculated from resource use expected using recommendations for follow-up of women with early breast cancer [36]. General practitioner visits, specialist visits, and mammograms were valued using Medicare Benefit Scheme item pricing. The costs of treating a recurrence were derived from Verry et al. (2012) [35]. Women who experienced a recurrence were assumed to complete treatment in a single one-year cycle. Healthcare costs for end-of-life care differed depending on if women died of breast cancer or other diseases reported in an Australian study by Reeves et al. (2017) [37]. The intervention was not expected to influence primary care and adjuvant treatments and these costs were not included.

Out-of-pocket costs were estimated for follow-up treatment and lost income for patients and their families while recovering from breast cancer or experiencing a recurrence. Productivity losses were estimated by hours of time off work from a recent Australian report [38] and adjusted for female labour force participation rates and age. Average Australian salaries for women were applied to estimate the costs [40] and the proportion of women of working age who work incorporating those who work partand full-time hours. Productivity lost was also included for women who died prematurely from breast cancer, up to the retirement age of 65 years, based on Australian estimates by Carter et al. (2016) [39].

\subsection{Analyses}

The main outcomes were mean costs, QALYs and life-years calculated from 5000 Monte Carlo simulations. An incremental cost-effectiveness ratio (ICER) was calculated as the difference in costs of the two strategies divided by the difference in QALYs or life years. An annual discount rate of $5 \%$ was applied to costs, QALYs and life-years as recommended by the Australian Government for outcomes extending beyond one year [43]. A half-cycle correction was applied to outcomes in the initial and 
final cycles as transitions could have occurred in the middle of each cycle. Costs were adjusted for inflation where necessary to 2019/2020 Australian dollars.

One-way sensitivity analyses were performed to calculate the ICERs using plausible variation in input values, re-running simulations 5000 times for each variable change. The $95 \%$ confidence intervals (CIs) of input values were used where available or $\pm 10 \%$ variation in mean values. Probabilistic sensitivity analyses were undertaken to assess the likelihood of the exercise intervention being cost-effective, considered at a willingness-to-pay threshold of AU\$50,000 per QALY gain. Input variables were assigned beta, gamma, and log normal distributions where appropriate (Supplementary File). To obtain the 95\% uncertainty interval (UI), we used the percentile method, ranking the incremental costs, QALYs, and ICERs and removing the top and bottom 2.5\%. Analyses were performed in TreeAge Pro Healthcare 2020 R2.1 software.

\section{Results}

Over the cohort's remaining life, the mean cost for the exercise group was AU $\$ 281,445$ (95\% UI $\$ 137,890$, \$372,701) compared with AU $\$ 274,035$ (95\% UI \$135,309, \$362,044) for the usual care group (Table 2). The corresponding QALYs were 10.97 (95\%UI 8.56, 12.93) for the exercise group and 10.63 (95\%UI 8.26, 12.51) for usual care. The incremental cost per QALY gained for the exercise intervention was AU $\$ 21,247$ (95\%UI Dominant, \$31,398) (Table 2). The likelihood that the exercise intervention was cost-effective was $93.0 \%$ (Figures 2 and 3 ).

Table 2. Main results for costs $\$ A U$, quality-adjusted life years and life-years.

\begin{tabular}{ccccc}
\hline & Exercise & Usual Care & Incremental & 95\% UI \\
\hline Mean & Mean & Difference & \\
QALYs & $\$ 281,445$ & $\$ 274,035$ & $\$ 7409$ & Cost-saving, $\$ 16,275$ \\
Life-years & 10.97 & 10.63 & 0.35 & $0.20,0.52$ \\
Incremental cost per QALY & 25.64 & 24.82 & 0.82 & $0.39,1.4$ \\
Incremental cost per & - & - & $\$ 21,247$ & Dominant $^{1}, \$ 31,398$ \\
life-year saved & - & - & $\$ 8894$ & Dominant $^{1}, \$ 11,769$ \\
\hline
\end{tabular}

Dominant means cost saving and higher health effects. UI = uncertainty interval, QALY = quality-adjusted life year.

${ }^{1}$ Dominant means the exercise group resulted in cost savings and improved QALYs or life years.

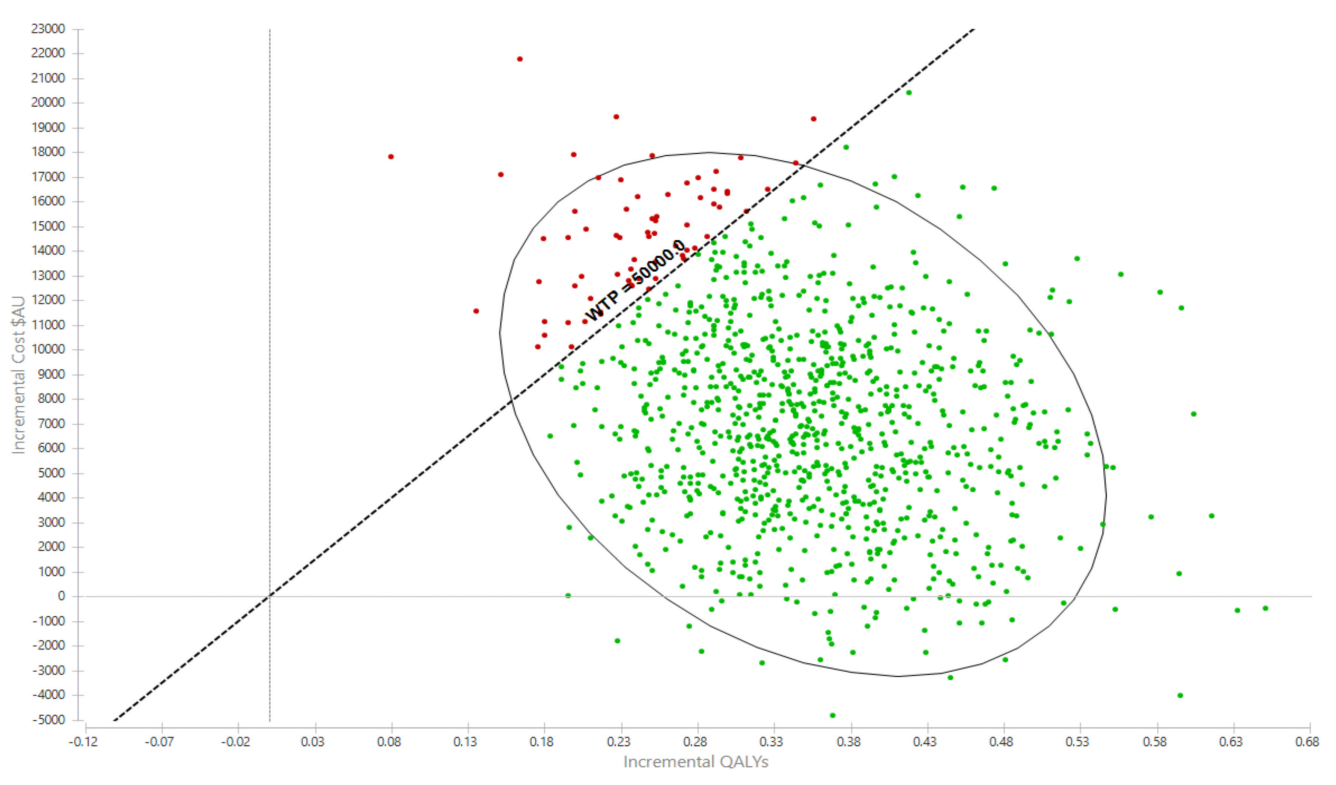

Figure 2. Probabilistic sensitivity analyses, incremental cost per QALY gain scatterplot. AU\$ = Australian dollars, QALY = quality-adjusted life year. 
Interpretation: Each dot represents an incremental cost and incremental life year pairing using the assigned distributions around each model parameter, selected randomly during 5000 iterations. Dots falling to the right of the diagonal line (the willingness-to-pay threshold of AU $\$ 50,000$ per quality-adjusted life year gained) are cost-effective. The proportion of simulations cost-effective is $93.0 \%$. The oval is the $95 \%$ ellipse and represents the $95 \%$ uncertainty interval.

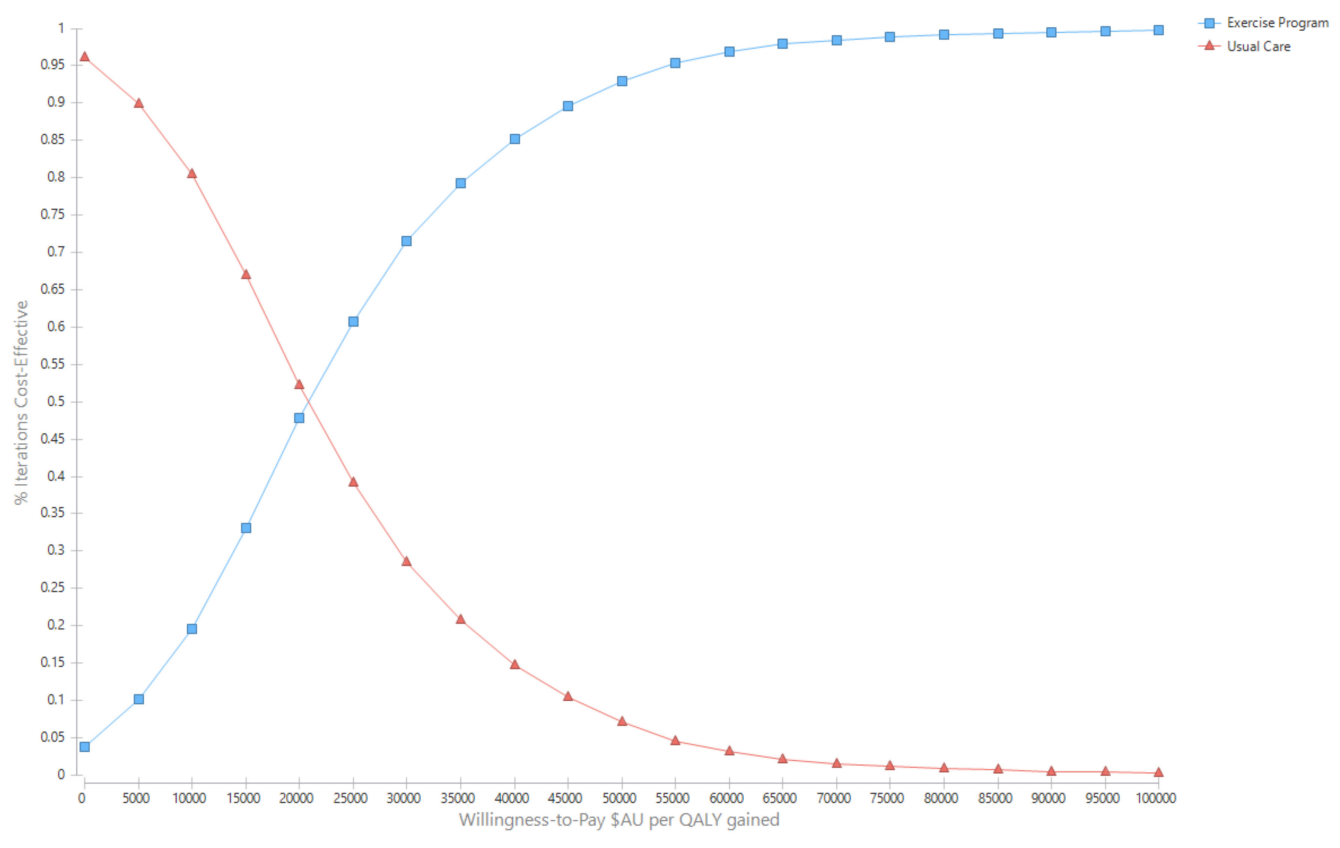

Figure 3. Cost-effectiveness acceptability curves.

Results for the outcome 'life-years saved' were more cost-effective than using QALYs as the outcome. The incremental cost per life year saved was \$8894 (95\% UI Dominant, \$11,769) with a 99.4\% probability of being cost effective.

The key factors influencing the results are the risk of breast cancer recurrence, patient out-of-pocket costs, and patient age. Modifications to risk of recurrence in the exercise and usual care groups was the only factor that influenced the incremental cost per QALY ratio in a meaningful way (Figure 4). Specifically, when the probability of recurrence in the exercise group was high $(0.0125)$, the potential cost-effectiveness of the intervention is less (that is, would no longer be considered cost-effective). In contrast, when probability of recurrence is higher than what was defined in the foundation model (which is likely), the intervention becomes particularly cost-effective (that is, would reduce the incremental cost per QALY ratio by $\$ 23,596$ over the long term) (Figure 4). While modification to other factors including age at diagnosis, non-breast cancer mortality, and health-related quality of life also influenced findings (making exercise more or less cost-effective), the incremental cost-effectiveness ratios remained below the cost-effective threshold of $\$ 50,000$ (Figure 4). 


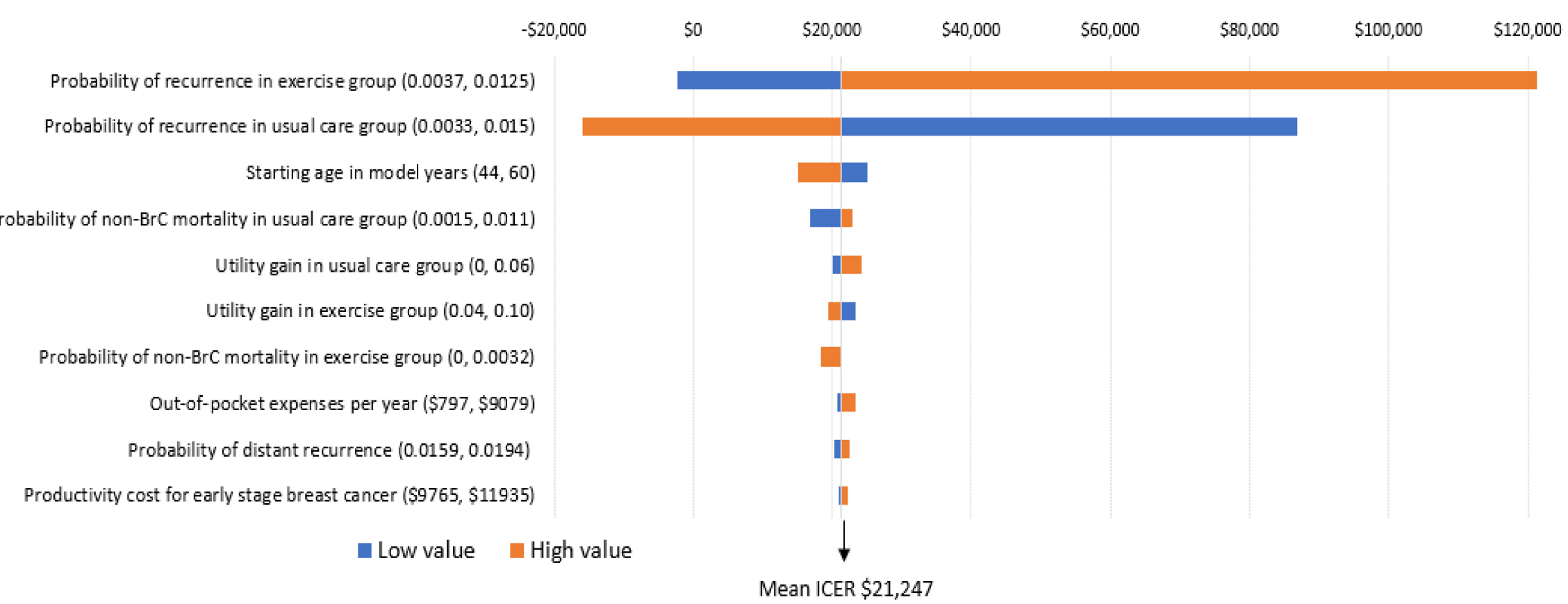

Figure 4. One-way sensitivity analyses; incremental cost per QALY gain. 
Interpretation: The importance of each variable on the ICER (incremental cost per QALY ratio) is presented from top to bottom. The high and low values for each variable are presented in brackets. The tails of each bar represent the maximum and minimum ICER for each variable. The vertical line indicates the ICER from the base case $(\$ 21,247)$ to provide a reference for the changes. Each high and low value was altered and 5000 simulations re-run to obtain these ICERs.

\section{Discussion}

Our analyses show that a tailored exercise intervention for women during and beyond treatment for early stage breast cancer is likely to be cost-effective in the Australian healthcare setting. That is, the benefits associated with exercise intervention were sufficient to warrant their additional costs. Our analysis included wider social costs accounting for time off work by patients and/or their carers and out-of-pocket costs for families, which reflect a broad view of the true costs of breast cancer. We took a conservative view for benefits by assessing QALYs and life years saved and made no assumptions about sustained quality of life or longevity after the 8-year follow-up period ended.

The benefits for women in the exercise group may be explained through improved health-related quality of life, improved progression-free survival or from higher overall survival, which the EfH trial suggests are robust long-term outcomes of exercise. Evidence shows that exercise programs are beneficial across all these effects [8,13]. The 8-year follow-up data from the EfH trial demonstrated that mixed-type, moderate-intensity exercise was favorable to disease recurrence and overall survival outcomes, irrespective of age, stage of disease, presence of other comorbidities, or body mass index [18]. Although the EfH trial was not powered or designed to detect significant group differences in these survival outcomes, evidence from meta-analyses also support these findings (overall survival hazard ratio 0.59 , 95\%CI: $0.53-0.65, p<0.01$ across 6 cohort studies) [13,44]. Overall survival is protected by regular exercise through improved physiological functioning in the immune, hormone, musculoskeletal, and cardiovascular systems and through protective psychological effects in reducing anxiety and depression [12-15]. For women who have had chemotherapy or adjuvant radiotherapy, exercise can counteract the late effects of cardiotoxicity caused by damage to the chest and heart [45]. In addition, since most women in the study had at least one or more comorbidities at baseline, it is plausible that women who were exercising in the EfH trial had improvements in cardiovascular disease, osteoporosis, and Type 2 diabetes.

Six cost-effectiveness studies have been reported on exercise programs for women following breast cancer diagnosis [20-25]. It is difficult to make clear comparisons across the studies due to their different health systems, and for interventions with different components, durations, and exercise intensities. Nonetheless, these studies show mixed results but all showed reasonably low costs for the exercise interventions with one having cost savings [24]. In general, overall healthcare costs between the intervention and control groups were similar, meaning in most cases, the intervention paid for itself by creating healthcare cost-offsets. Although three studies found favourable cost-effective findings [23-25], those with more questionable economic efficiency showed insufficient benefit for exercise rather than being costly. There are several possible explanations for exercise having relatively small benefits in cost-effectiveness studies. Despite the wide support and recommendation by decision-making bodies to use QALYs in cost-effectiveness analysis, this metric may be insensitive when marked survival differences are not expected, or when symptom severity or change in severity of symptoms are unlikely to be reasonably captured using the EuroQoL-5D health utility tool. In this particular population, women have early stage breast cancer with good survival prospects and similar quality of life post-breast cancer (compared with age-matched norms) making it more difficult to demonstrate QALY gains. Furthermore, the potential for contamination in control groups, as was observed in two studies [20,22], also influences cost-effectiveness findings in the conservative direction (that is, makes it less likely to find an exercise intervention cost-effective). Of note, it is also known in the wider exercise oncology base, that participants who have been physically active pre-cancer are more likely to participate in exercise trials and that therefore participants randomized to the control group of an exercise oncology 
trial are more likely to engage in exercise of their own accord than those who do not participate in exercise trials [46]. In another study, the intervention consisted of a low dose intervention (i.e., 3 contacts over 12 months) which is likely to be insufficient to show effectiveness [21]. Taken together with their substantial heterogeneity, and conservative approaches to valuation, these studies suggest that exercise interventions are beneficial across various outcome measures and low cost to society. These findings potentially highlight the inadequacy of the current public reimbursement system in Australia, which caps the number of sessions with an allied health professional over 12 months at five, with these sessions needing to be shared among all allied health professionals.

In broad terms, physical inactivity incurs a massive economic cost in Australia, estimated up to $\$ 850$ million in healthcare expenditure and $\$ 15.6$ billion in lost production, per year [47]. Despite this, public health investment in disease prevention through promotion of healthy behaviors is challenging when upfront outlays are needed and expected health benefits are delayed. However, prevention is an essential component of an effective health system. Compared with other developed countries whose governments spend $2-4 \%$ of health expenditure on prevention, Australia has below-average prevention investment (1.5\%) [48]. Worryingly for many countries, a large proportion of spending in prevention is spent on less cost-effective measures [48] and economic evaluations are required to inform decision-makers of the "best buys." For example, evidence on national breast cancer screening generally confirms its cost-effectiveness, and would be further improved with risk-based approaches that also reduce over-diagnosis, but there is less consensus on the cost-effectiveness of general health check-ups [48].

The strengths of this work include using high-quality randomized controlled trial evidence (with intention-to-treat analysis, complete follow-up data), robust data from large recent studies and, the model's lifetime analytical horizon. This model has likely underestimated the benefits to women's health from the exercise intervention, particularly relating to reductions in poor health effects from comorbid conditions. In addition, potential benefits from exercise may have arisen from the reduced need for primary care, reduced risk of dose reductions and delays associated with adjuvant treatment, and reduced risk of new breast cancer. Therefore, we consider our analysis to be conservative. A societal viewpoint of costs were taken to capture the full implications of productivity losses and family disruptions. Limitations of this study include the reliance on international data estimates when Australian epidemiological statistics were not available and the use of breast cancer recurrence estimates (based on small numbers) from the $\mathrm{EfH}$ trial. Whether the impact of chemotherapy completion has positive longer-term benefits also requires more evidence to confirm this definitively and this model could be updated in due course.

As with many health promotion programs, often the most disadvantaged groups are more vulnerable to poorer health outcomes while also being the least likely to participate. One element of socioeconomic disadvantage is living in rural or remote locations with less access to healthcare services. The evaluated exercise program was delivered remotely to women residing rurally via telehealth, which had the dual benefits of reaching a broad population geographically while keeping patient out-of-pocket costs and time off work low. Further research could assess whether telehealth platforms are feasible for follow-up maintenance programs and for delivering care to other cancer populations.

\section{Conclusions}

The EfH tailored exercise intervention for women after breast cancer diagnosis is cost-effective compared with usual care. Investing in resources to facilitate prescribed regular exercise in this population should be a priority for cancer service providers.

Supplementary Materials: The following are available online at http://www.mdpi.com/1660-4601/17/22/8608/s1. Table S1. Detail of intervention delivery costs. Table S2: Productivity losses, end-of-life healthcare costs, and out-of-pocket costs. Table S3: Costs for recurrence of breast cancer. Table S4: Follow-up schedule. Table S5: Probability of local and distant recurrence. Table S6: Probability of locoregional disease progressing to distant disease. Table S7: Survival rates from breast cancer by stage. Table S8: Mortality and recurrence rates for exercise and usual care groups (source EfH trial). Table S9: Probability of death by age (female). 
Author Contributions: Conceptualization, L.G.G. and S.C.H.; methodology, L.G.G.; formal analysis, L.G.G.; data management S.C.H. and R.R.S.; writing—original draft preparation, L.G.G.; writing-review and editing, all authors; supervision, S.C.H.; project administration, S.C.H. and R.R.S.; funding acquisition, S.C.H., C.P., E.G.E., J.B., S.C.H., C.S. All authors have read and agreed to the published version of the manuscript.

Funding: This research was funded by the National Breast Cancer Foundation, Australia. Author S.C.H. was supported by a National Breast Cancer Foundation Fellowship during the period of the conduct of the project.

Acknowledgments: We wish to thank the women in the study who generously made time to participate in this project.

Conflicts of Interest: The authors declare no conflict of interest.

\section{References}

1. International Agency for Research on Cancer (WHO). Global Cancer Observatory; IARC: Lyon, France, 2020.

2. Australian Institute of Health and Welfare (AIHW). Cancer in Australia: In Brief 2019; AIHW: Canberra, Australia, 2019.

3. Australian Institute of Health and Welfare (AIHW). Cancer in Australia 2019 Cat. No. CAN 123. Available online: https://meteor.aihw.gov.au/content/index.phtml/itemId/698954 (accessed on 20 October 2020).

4. Heer, E.; Harper, A.; Escandor, N.; Sung, H.; McCormack, V.; Fidler-Benaoudia, M.M. Global burden and trends in premenopausal and postmenopausal breast cancer: A population-based study. Lancet Glob. Health 2020, 8, e1027-e1037. [CrossRef]

5. Kenyon, M.; Mayer, D.K.; Owens, A.K. Late and long-term effects of breast cancer treatment and surveillance management for the general practitioner. J. Obstet. Gynecol. Neonatal. Nurs. 2014, 43, 382-398. [CrossRef]

6. Islam, T.; Dahlui, M.; Majid, H.A.; Nahar, A.M.; Mohd Taib, N.A.; Su, T.T. Factors associated with return to work of breast cancer survivors: A systematic review. BMC Public Health 2014, 14 (Suppl. S3), S8. [CrossRef]

7. Demark-Wahnefried, W.; Schmitz, K.H.; Alfano, C.M.; Bail, J.R.; Goodwin, P.J.; Thomson, C.A.; Bradley, D.W.; Courneya, K.S.; Befort, C.A.; Denlinger, C.S.; et al. Weight management and physical activity throughout the cancer care continuum. CA Cancer J. Clin. 2018, 68, 64-89. [CrossRef]

8. Cormie, P.; Adams, D.; Atkinson, M.; Bucci, L.; Cust, A.E.; Eakin, E.; McCarthy, A.L.; Murnane, A.; Quinn, S.; Hayes, S.C. Exercise as part of routine cancer care. Lancet Oncol. 2018, 19, e432. [CrossRef]

9. Cormie, P.; Atkinson, M.; Bucci, L.; Cust, A.; Eakin, E.; Hayes, S.; McCarthy, S.; Murnane, A.; Patchell, S.; Adams, D. Clinical Oncology Society of Australia position statement on exercise in cancer care. Med. J. Aust. 2018, 209, 184-187. [CrossRef] [PubMed]

10. Hayes, S.C.; Newton, R.U.; Spence, R.R.; Galvão, D.A. The Exercise and Sports Science Australia position statement: Exercise medicine in cancer management. J. Sci. Med. Sport. 2019, 22, 1175-1199. [CrossRef] [PubMed]

11. Patel, A.V.; Friedenreich, C.M.; Moore, S.C.; Hayes, S.C.; Silver, J.K.; Campbell, K.L.; Winters-Stone, K.; Gerber, L.H.; George, S.M.; Fulton, J.E.; et al. American College of Sports Medicine Roundtable Report on Physical Activity, Sedentary Behavior, and Cancer Prevention and Control. Med. Sci. Sports Exerc. 2019, 51, 2391-2402. [CrossRef]

12. Friedenreich, C.M.; Stone, C.R.; Cheung, W.Y.; Hayes, S.C. Physical Activity and Mortality in Cancer Survivors: A Systematic Review and Meta-Analysis. JNCI Cancer Spectr. 2020, 4, pkz080. [CrossRef] [PubMed]

13. Lahart, I.M.; Metsios, G.S.; Nevill, A.M.; Carmichael, A.R. Physical activity, risk of death and recurrence in breast cancer survivors: A systematic review and meta-analysis of epidemiological studies. Acta Oncol. 2015, 54, 635-654. [CrossRef]

14. Schmid, D.; Leitzmann, M.F. Association between physical activity and mortality among breast cancer and colorectal cancer survivors: A systematic review and meta-analysis. Ann. Oncol. 2014, 25, 1293-1311. [CrossRef] [PubMed]

15. Spei, M.E.; Samoli, E.; Bravi, F.; La Vecchia, C.; Bamia, C.; Benetou, V. Physical activity in breast cancer survivors: A systematic review and meta-analysis on overall and breast cancer survival. Breast. 2019, 44, 144-152. [CrossRef] [PubMed] 
16. Eakin, E.G.; Lawler, S.P.; Winkler, E.A.; Hayes, S.C. A randomized trial of a telephone-delivered exercise intervention for non-urban dwelling women newly diagnosed with breast cancer: Exercise for health. Ann. Behav. Med. 2012, 43, 229-238. [CrossRef] [PubMed]

17. Hayes, S.C.; Rye, S.; Disipio, T.; Yates, P.; Bashford, J.; Pyke, C.; Saunders, C.; Battistutta, D.; Eakin, E. Exercise for health: A randomized, controlled trial evaluating the impact of a pragmatic, translational exercise intervention on the quality of life, function and treatment-related side effects following breast cancer. Breast Cancer Res. Treat. 2013, 137, 175-186. [CrossRef] [PubMed]

18. Hayes, S.C.; Steele, M.L.; Spence, R.R.; Gordon, L.; Battistutta, D.; Bashford, J.; Pyke, C.; Saunders, C.; Eakin, E. Exercise following breast cancer: Exploratory survival analyses of two randomised, controlled trials. Breast Cancer Res. Treat. 2018, 167, 505-514. [CrossRef]

19. Khan, K.A.; Mazuquin, B.; Canaway, A.; Petrou, S.; Bruce, J. Systematic review of economic evaluations of exercise and physiotherapy for patients treated for breast cancer. Breast Cancer Res. Treat. 2019, 176, 37-52. [CrossRef]

20. Gordon, L.G.; DiSipio, T.; Battistutta, D.; Yates, P.; Bashford, J.; Pyke, C.; Eakin, E.; Hayes, S.C. Cost-effectiveness of a pragmatic exercise intervention for women with breast cancer: Results from a randomized controlled trial. Psychooncology. 2017, 26, 649-655. [CrossRef]

21. Haines, T.P.; Sinnamon, P.; Wetzig, N.G.; Lehman, M.; Walpole, E.; Pratt, T.; Smith, A. Multimodal exercise improves quality of life of women being treated for breast cancer, but at what cost? Randomized trial with economic evaluation. Breast Cancer Res. Treat. 2010, 124, 163-175. [CrossRef]

22. May, A.M.; Bosch, M.J.; Velthuis, M.J.; van der Wall, E.; Steins Bisschop, C.N.; Los, M.; Erdkamp, F.; Bloemendal, H.J.; de Roos, M.A.; Verhaar, M.; et al. Cost-effectiveness analysis of an 18-week exercise programme for patients with breast and colon cancer undergoing adjuvant chemotherapy: The randomised PACT study. BMJ Open. 2017, 7, e012187. [CrossRef]

23. Mewes, J.C.; Steuten, L.M.; Ijzerman, M.J.; van Harten, W.H. Effectiveness of multidimensional cancer survivor rehabilitation and cost-effectiveness of cancer rehabilitation in general: A systematic review. Oncologist 2012, 17, 1581-1593. [CrossRef]

24. Perrier, L.; Foucaut, A.M.; Morelle, M.; Touillaud, M.; Kempf-Lépine, A.S.; Heinz, D.; Gomez, F.; Meyrand, R.; Baudinet, C.; Berthouze, S.; et al. Cost-effectiveness of an exercise and nutritional intervention versus usual nutritional care during adjuvant treatment for localized breast cancer: The PASAPAS randomized controlled trial. Support. Care Cancer. 2020, 28, 2829-2842. [CrossRef] [PubMed]

25. van Waart, H.; van Dongen, J.M.; van Harten, W.H.; Stuiver, M.M.; Huijsmans, R.; Hellendoorn-van Vreeswijk, J.; Sonke, G.S.; Aaronson, N.K. Cost-utility and cost-effectiveness of physical exercise during adjuvant chemotherapy. Eur. J. Health Econ. 2018, 19, 893-904. [CrossRef] [PubMed]

26. Husereau, D.; Drummond, M.; Petrou, S.; Carswell, C.; Moher, D.; Greenberg, D.; Augustovski, F.; Briggs, A.H.; Mauskopf, J.; Loder, E. Consolidated Health Economic Evaluation Reporting Standards (CHEERS) statement. Value Health. 2013, 16, e1-e5. [CrossRef] [PubMed]

27. Philips, Z.; Bojke, L.; Sculpher, M.; Claxton, K.; Golder, S. Good practice guidelines for decision-analytic modelling in health technology assessment: A review and consolidation of quality assessment. Pharmacoeconomics 2006, 24, 355-371. [CrossRef] [PubMed]

28. Hayes, S.; Rye, S.; Battistutta, D.; Yates, P.; Pyke, C.; Bashford, J.; Eakin, E. Design and implementation of the Exercise for Health trial-A pragmatic exercise intervention for women with breast cancer. Contemp. Clin. Trials. 2011, 32, 577-585. [CrossRef] [PubMed]

29. Wu, X.; Baig, A.; Kasymjanova, G.; Kafi, K.; Holcroft, C.; Mekouar, H.; Carbonneau, A.; Bahoric, B.; Sultanem, K.; Muanza, T. Pattern of Local Recurrence and Distant Metastasis in Breast Cancer By Molecular Subtype. Cureus 2016, 8, e924. [CrossRef]

30. Wapnir, I.L.; Anderson, S.J.; Mamounas, E.P.; Geyer, C.E., Jr.; Jeong, J.H.; Tan-Chiu, E.; Fisher, B.; Wolmark, N. Prognosis after ipsilateral breast tumor recurrence and locoregional recurrences in five National Surgical Adjuvant Breast and Bowel Project node-positive adjuvant breast cancer trials. J. Clin. Oncol. 2006, 24, 2028-2037. [CrossRef]

31. Anderson, S.J.; Wapnir, I.; Dignam, J.J.; Fisher, B.; Mamounas, E.P.; Jeong, J.H.; Geyer, C.E., Jr.; Wickerham, D.L.; Costantino, J.P.; Wolmark, N. Prognosis after ipsilateral breast tumor recurrence and locoregional recurrences in patients treated by breast-conserving therapy in five National Surgical Adjuvant Breast and Bowel Project protocols of node-negative breast cancer. J. Clin. Oncol. 2009, 27, 2466-2473. [CrossRef] 
32. Australian Bureau of Statistics. Life Tables, States, Territories and Australia, 2016-2018 Cat. No. 3302.0.55.001; Commonwealth of Australia: Canberra, Australia, 2019.

33. Surveillance, Epidemiology and End Results Program. Cancer Statistics, Cancer Stat. Facts: Female Breast Cancer; National Cancer Institute: Bethesda, Maryland, USA, 2020.

34. Witteveen, A.; Kwast, A.B.; Sonke, G.S.; MJ, I.J.; Siesling, S. Survival after locoregional recurrence or second primary breast cancer: Impact of the disease-free interval. PLoS ONE. 2015, 10, e0120832. [CrossRef]

35. Verry, H.; Lord, S.J.; Martin, A.; Gill, G.; Lee, C.K.; Howard, K.; Wetzig, N.; Simes, J. Effectiveness and cost-effectiveness of sentinel lymph node biopsy compared with axillary node dissection in patients with early-stage breast cancer: A decision model analysis. Br. J. Cancer. 2012, 106, 1045-1052. [CrossRef]

36. Cancer Australia. Clinical Practice Guidelines: Recommendations for Follow-Up for Women with Early Breast Cancer; Cancer Australia: Sydney, Australia, 2011.

37. Reeve, R.; Srasuebkul, P.; Langton, J.M.; Haas, M.; Viney, R.; Pearson, S.A. Health care use and costs at the end of life: A comparison of elderly Australian decedents with and without a cancer history. BMC Palliat. Care 2017, 17, 1. [CrossRef] [PubMed]

38. Deloitte Access Economics. Financial Impact of Breast Cancer in Australia. A report for Breast Cancer Network Australia; Deloitte Access Economics: Sydney, Australia, 2016.

39. Carter, H.E.; Schofield, D.J.; Shrestha, R. The Productivity Costs of Premature Mortality Due to Cancer in Australia: Evidence from a Microsimulation Model. PLoS ONE 2016, 11, e0167521. [CrossRef] [PubMed]

40. Australian Government. Employee Earnings and Hours, Australia Jan 2019; Australian Government: Canberra, Australia, 2019.

41. Paracha, N.; Thuresson, P.O.; Moreno, S.G.; MacGilchrist, K.S. Health state utility values in locally advanced and metastatic breast cancer by treatment line: A systematic review. Expert Rev. Pharm. Outcomes Res. 2016, 16, 549-559. [CrossRef] [PubMed]

42. Fleurence, R.L.; Hollenbeak, C.S. Rates and probabilities in economic modelling: Transformation, translation and appropriate application. Pharmacoeconomics 2007, 25, 3-6. [CrossRef]

43. Government, A. The Pharmaceutical Benefits Advisory Committee Guidelines Version 5.0; Australian Government: Canberra, Australia, 2016.

44. Ibrahim, E.M.; Al-Homaidh, A. Physical activity and survival after breast cancer diagnosis: Meta-analysis of published studies. Med. Oncol. 2011, 28, 753-765. [CrossRef]

45. Cavarretta, E.; Mastroiacovo, G.; Lupieri, A.; Frati, G.; Peruzzi, M. The Positive Effects of Exercise in Chemotherapy-Related Cardiomyopathy. Adv. Exp. Med. Biol 2017, 1000, 103-129. [CrossRef]

46. Spence, R.R.; DiSipio, T.; Schmitz, K.; Hayes, S. Is unsupervised exercise following breast cancer safe for all women? Int. J. Phys. Med. Rehabil. 2014, 2, 3. [CrossRef]

47. Crosland, P.; Ananthapavan, J.; Davison, J.; Lambert, M.; Carter, R. The economic cost of preventable disease in Australia: A systematic review of estimates and methods. Aust. N. Z. J. Public Health. 2019, 43, 484-495. [CrossRef]

48. Gmeinder, M.; Morgan, D.; Mueller, M. How Much Do OECD Countries Spend on Prevention? Organisation for Economic Co-operation and Development: Paris, France, 2017.

Publisher's Note: MDPI stays neutral with regard to jurisdictional claims in published maps and institutional affiliations.

(C) 2020 by the authors. Licensee MDPI, Basel, Switzerland. This article is an open access article distributed under the terms and conditions of the Creative Commons Attribution (CC BY) license (http://creativecommons.org/licenses/by/4.0/). 